\title{
Regional dopamine transporter gene expression in the substantia nigra from control and Parkinson's disease brains
}

Timothy J Counihan, John B Penney Jr

\begin{abstract}
Objective-To test the hypothesis that differential regional dopamine transporter (DAT) gene expression may underlie the selective vulnerability of certain nigral dopaminergic neurons in Parkinson's disease, DAT mRNA expression was examined in neuronal subpopulations of human postmortem ventral mesencephalon from patients with Parkinson's disease and controls.

Methods-Radioactive in situ hybridisation histochemistry using a polymerase chain reaction derived ribonucleotide probe for DAT was performed on sections of ventral mesencephalon from the brains of five donors with no history of neurological illness and from five patients with pathologically established Parkinson's disease. The number of silver grains overlying melanised neurons from the paranigral nucleus, dorsal and ventral tier, and pars lateralis of the substantia nigra pars compacta were compared with each other and to background labelling by using a one way factorial analysis of variance (ANOVA) with a significance level of $5 \%$.
\end{abstract}

Results-In control brains, there was intense DAT mRNA expression in the ventral midbrain with no significant difference in mRNA concentrations among the four regions studied. In the Parkinson's disease brains, there was an overall decrease in the intensity of DAT mRNA expression in the surviving dopaminergic neurons. There were no significant differences in signal between regions in either the control or parkinsonian brains. Conclusion-Taken together, these findings do not support the hypothesis that differential regional DAT gene expression underlies the selective vulnerability of certain nigral dopaminergic neurons in Parkinson's disease, as the vulnerable neurons of the substantia nigra pars compacta do not express more DAT mRNA than the resistant paranigral neurons. (F Neurol Neurosurg Psychiatry 1998;65:164-169)

Keywords: mesencephalon; in situ hybridisation; parkinsonism; amine transporter

Penney, Neurology Research, Warren 408, Massachusetts General Hospital, Boston, MA 02114, USA. Telephone 001617726 5570; fax 001 6177241480 .

Received 1 September 1997 and in revised form

5 January 1998

Accepted 13 January 1998

The pathological hallmark of Parkinson's disease is degeneration of dopaminergic neurons of the ventral mesencephalon. The degenerative process is not uniform across all dopaminergic neurons, but is characterised by a selective vulnerability of specific subpopulations, whereas other neuronal groups are relatively spared..$^{1-3}$ Neurons of the ventral tier of the substantia nigra pars compacta (SNpc) seem to be the most vulnerable, followed by those of the dorsal tier, with relative sparing of the medial substantia nigra and pars lateralis. ${ }^{3}$

The dopamine transporter DAT, a member of a large family of $\mathrm{Na}+\mathrm{Cl}-$ dependent transporters, ${ }^{4}$ is thought to control the synaptic activity of released dopamine by rapid reuptake of the neurotransmitter into presynaptic terminals. ${ }^{5}$ It has received increasing attention recently because of its role as a target for psychoactive drugs, ${ }^{6}$ as well as acting as a gate for several neurotoxins that destroy dopaminergic neurons, including 6-hydroxydopamine and 1,2,3,6-tetrahydro1-methyl-4-phenylpyridine (MPTP). ${ }^{78}$ The toxic metabolite of MPTP, 1-methyl-4phenylpyridinium $\left(\mathrm{MPP}^{+}\right)$, enters dopaminergic neurons through the DAT, thereafter accumulating in the mitochondria where it inhibits complex I of the electron transport chain. Because of the similarities between MPTP toxicity and idiopathic Parkinson's disease, ${ }^{9}$ it has been suggested that the dopaminergic cell loss might result from exposure to a similar exogenous substance. Previous studies in rodents $^{10-12}$ and humans ${ }^{13-17}$ have identified a robust expression of the DAT mRNA in neurons of the ventral mesencephalon, with the most intense expression in $\mathrm{SNpc}$ neurons. Neurons of the medial substantia nigra nuclei have been variously reported as having either more, ${ }^{16}$ or less ${ }^{10}{ }^{15}$ DAT gene expression, compared with SNpc neuronal groups, a discrepancy that may be explained in part by the lack of consensus in the literature regarding the cytoarchitectonic structure comprising the A10 cell group of dopaminergic neurons in human brain. ${ }^{18}$

Whereas hybridisation studies of DAT gene expression in Parkinson's disease ${ }^{15} 16$ have shown a marked reduction in DAT signal in the surviving dopaminergic neurons, quantitative studies considering DAT gene expression in specific neuronal subpopulations of ventral mesencephalon in control and Parkinson's disease brains are lacking.

We therefore analysed regional DAT gene expression in selected neuronal subpopulations of the ventral mesencephalon in control and Parkinson's disease brains, using in situ hybridisation histochemistry. 
Table 1 Clinical characteristics of patients and postmortem data

\begin{tabular}{lllll}
\hline Patient & Sex & Age & $\begin{array}{l}\text { Tissue } \\
p H\end{array}$ & $\begin{array}{l}\text { Postmortem } \\
\text { interval (h) }\end{array}$ \\
\hline Control: & & & & \\
$\quad$ Coronary disease & $\mathrm{M}$ & 61 & 6.6 & 23 \\
$\quad$ Heart failure & $\mathrm{F}$ & 87 & 5.9 & 11 \\
Carcinoma larynx & $\mathrm{M}$ & 68 & 6.3 & 15 \\
Myocardial infarct & $\mathrm{F}$ & 72 & 6.6 & 11 \\
$\quad$ Pneumonia & $\mathrm{F}$ & 90 & 6.6 & 19 \\
Parkinson's disease: & & & & \\
1 & $\mathrm{M}$ & 88 & 6.3 & 23 \\
2 & $\mathrm{M}$ & 80 & 6.3 & 23 \\
3 & $\mathrm{M}$ & 78 & 6.3 & 22 \\
4 & $\mathrm{M}$ & 78 & 6.1 & 24 \\
$5^{\star}$ & $\mathrm{F}$ & 80 & 6.0 & 9 \\
\hline
\end{tabular}

^Also had extranigral Lewy bodies.

\section{Materials and methods}

POSTMORTEM SPECIMENS

Tissue blocks from the brains of five donors with no history of neurological illness and from five patients with pathologically established Parkinson's disease were obtained from the Brain Tissue Resource Center, McLean hospital, Belmont, MA, USA, and the Michigan Brain Disease Program, Ann Arbor, MI, USA. Table 1 summarises the clinical characteristics. The subjects were matched for age (71.2 (SD 12.3) years versus 81 (SD 7.3), $\mathrm{p}<0.14$, for controls and Parkinson's disease, respectively) and postmortem interval (19.8 (SD 7.3) hours versus 20.8 (SD 6) hours). All diseased patients were taking levodopa at the time of death except for patient 5, who had discontinued levodopa three months before death. None of the patients had a history of dementia or prominent psychosis.

At postmortem examination, the brain was divided in the mid-sagittal plane and the brainstem was cut transversely at the level of the exit of the oculomotor nerve. A single $5 \mathrm{~mm}$ block from one side was quick-frozen in chilled isopentane and stored at $-70^{\circ} \mathrm{C}$ until use. Only brains with $\mathrm{pH}>5.8$ were used, as tissue $\mathrm{pH}$ has been found to be strongly correlated with mRNA preservation in human postmortem brain. ${ }^{19}$

GENERATION OF PROBES

A DNA template for synthesising an RNA probe for $\mathrm{DAT}^{20}$ was constructed using a polymerase chain reaction (PCR) strategy that is a modification of the method of Sitzman et $a l .{ }^{21}$ Complementary DNA was prepared from whole human brain poly A+ mRNA (Clontech, Palo Alto, CA, USA) using a reverse transcriptase reaction kit and oligodeoxythymidine primers (Promega, Madison, WI, USA) as directed by the manufacturer. Software analysis using Oligo 4.0 (National Biosciences Inc, Plymouth, MN, USA) was used to optimise primer design. Primers were purchased from Life Technologies (Gaithersburg, MD, USA). Outer primers consisting of 20mers specific for bases 1991-2010 and 2430-2449 of the targeted gene segment and amplifying a segment of 458 base pairs, were used to amplify DNA obtained by reverse transcription of human brain poly A mRNA (Promega). The product of this amplification served as starting material for amplification with an inner primer pair specific for bases 2025-2043 and 2421-2420 coupled to 20mers specific for the promoters of the RNA polymerases SP6 and T7 respectively. The final template size was 415 base pairs, recognising all three forms of the human DAT mRNA. ${ }^{5}$

LABELLING OF RNA PROBE

A radioactively labelled cRNA probe to DAT was synthesised by in vitro transcription using SP6 RNA polymerase (Promega) as previously described. ${ }^{22}$

NORTHERN BLOT ANALYSIS

Northern blot analysis was used to evaluate the specificity of the DAT RNA probe. The probe for northern analysis was prepared from PCR products by random primer extension, using the Prime-a-Gene system (Promega) and $\left[{ }^{32} \mathrm{P}\right] \mathrm{dCTP}$. A commercially available human brain northern blot (MTN Blot III, Clontech), was hybridised with labelled probe in ExpressHyb hybridisation solution (Clontech) for 1 hour. After several posthybridisation washes, the blot was then exposed to $x$ ray film (Hyperfilm-MP, Amersham, Arlington Heights, IL, USA) at $-70^{\circ} \mathrm{C}$ with an intensifying screen for 2 hours.

IN SITU HYBRIDISATION:

In situ hybridisation histochemistry was performed according to a standard protocol. ${ }^{22}$ Briefly, sections $(12 \mu \mathrm{m})$ of human midbrain were cut by using a cryostat and stored at $-70^{\circ} \mathrm{C}$. The slides were warmed to room temperature, fixed in $4 \%$ paraformaldehyde in 0.1 $\mathrm{M}$ phosphate buffer, $\mathrm{pH} 7.4$, washed in three changes of $0.1 \mathrm{M}$ phosphate buffered saline (PBS), acetylated in $0.1 \mathrm{M}$ triethanolamine, pH 8.0 with $0.25 \%$ acetic anhydride, rinsed in PBS, dehydrated through graded ethanol solutions, and delipidated in chloroform. Sections were then hybridised at $50^{\circ} \mathrm{C}$ for 4 hours. The quantity of radioactive probe included was adjusted to achieve $150000 \mathrm{cpm} / \mu \mathrm{l}$ hybridisation buffer. After hybridisation, sections were washed in sodium chloride-sodium citrate (SSC), then treated with RNase A, rinsed in RNase buffer and washed twice more in SSC. Film autoradiograms were prepared by apposing the slides to Hyperfilm b-Max (Amersham) for 3 to 7 days. Slides were then dipped in NTB-2 emulsion (Eastman Kodak, Rochester, NY, USA), stored at $4^{\circ} \mathrm{C}$, and developed at 4 weeks. Specificity of the hybridisation was monitored by the use of sense strand probes.

\section{ANALYSIS OF IN SITU HYBRIDISATION}

Emulsion autoradiograms were examined under brightfield illumination. Two sections from each of the 10 brains were included in the analyses. Sections were counter stained with haematoxylin and eosin to permit delineation of the neuronal profile. Regional boundaries of the substantia nigra were interpreted according to the scheme of McRitchie et $a l,{ }^{18}$ with additional reference to Olszewski and Baxter, ${ }^{23}$ Bogerts, ${ }^{24}$ and Gibb and Lees. ${ }^{1}$ Neurons from four cell groups of the ventral mesencephalon 

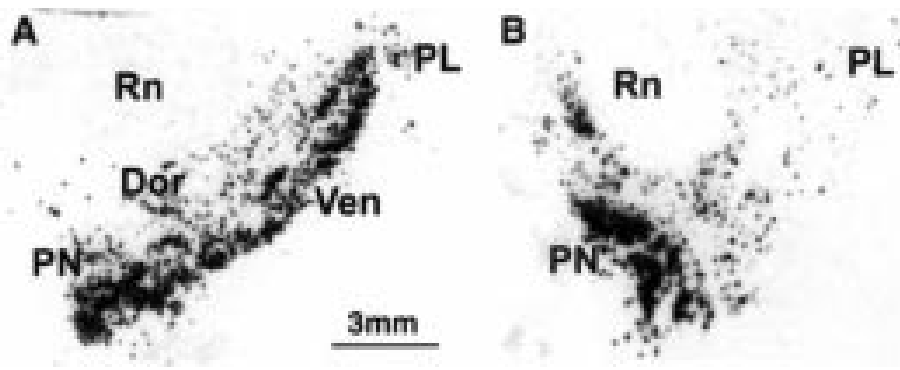

Figure 1 Film autoradiograms of a transverse section through human mesencephalon from a control $(A)$ and a Parkinson's disease $(B)$ brain, hybridised with a ${ }^{35} S$-labelled dopamine transporter (DAT) specific antisense RNAprobe. Regions analysed are indicated in panel A; paranigral nucleus (PN), dorsal (Dor), ventral (Ven), and pars lateralis (PL) of the substantia nigra pars compacta ( $S N p c$ ). The plane of section panel $B$ is more caudal than that in $A$, disclosing a more extensive paranigral nucleus. There is extensive loss of hybridisation signal in all parts of the SNpc in the Parkinson's disease brain, with relative preservation in the paranigral nucleus. $R n=$ red nucleus.

were analysed: the medial paranigral nucleus, delineated by the interspersing of the exiting oculomotor fibres, ${ }^{18}$ dorsal and ventral tier neuronal groups and the pars lateralis of the substantia nigra pars compacta. In the case of the Parkinson's disease brains where neuronal loss obscured the boundary between dorsal and ventral tier SNpc neurons, cells counted were considered as a single region $(\mathrm{SNpc})$.

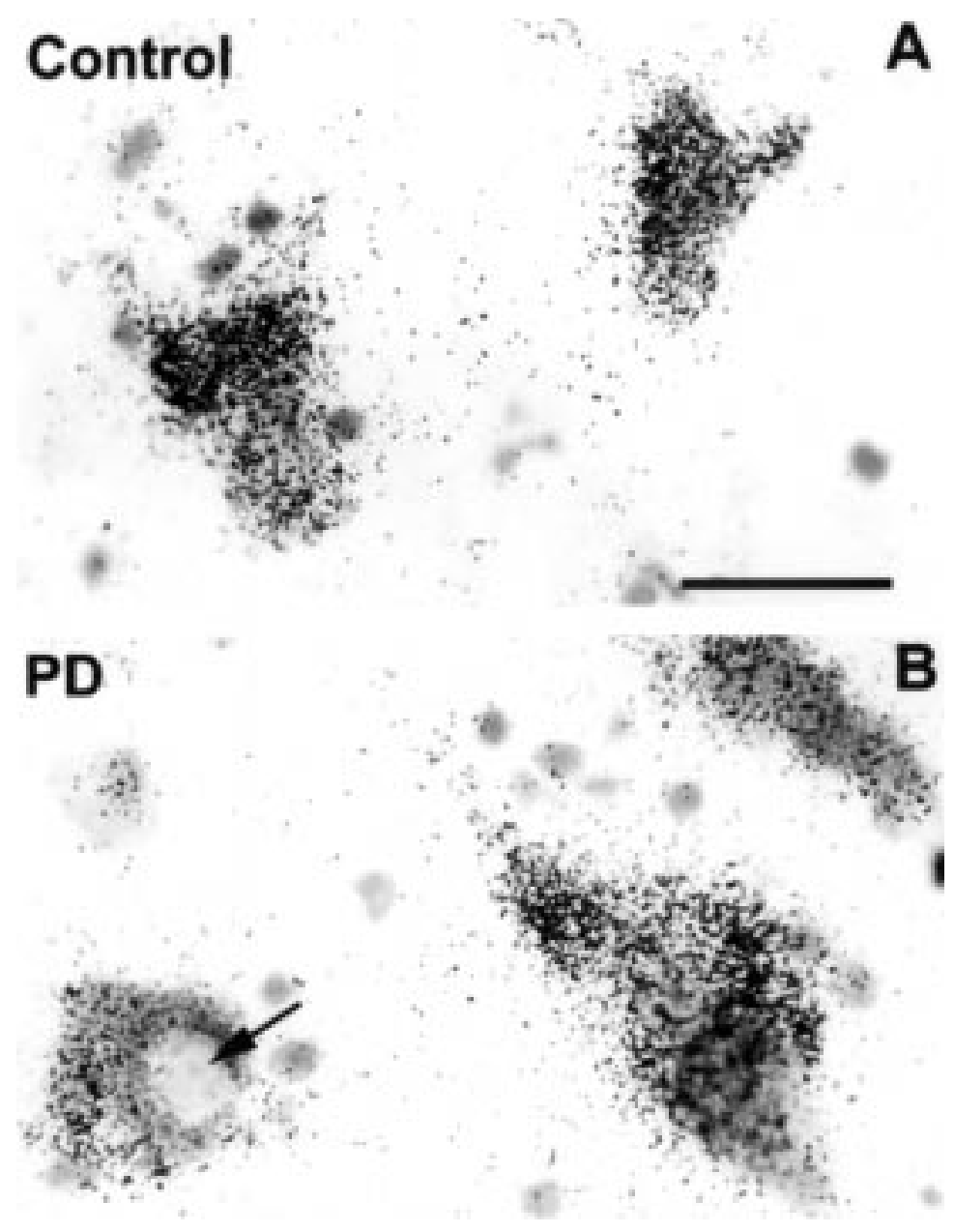

Figure 2 Expression of dopamine transporter (DAT) $m R N A$ in human control and Parkinson's disease midbrain dopaminergic neurons. Each panel shows autoradiographic silver grains produced by an ${ }^{35} S$-labelled $R N A$ probe for DAT. The arrow in panel $B$ indicates a Lewy body within the cytoplasm of a lightly labelled melanised neuron, compared with an adjacent intensely labelled neuron. Scale bar $=30 \mu \mathrm{m}$.
Quantitative analysis of DAT mRNA expression was performed with a computer assisted image analysis system (M1, Imaging Research, St. Catharine's, Ontario, Canada) ${ }^{22}$. The image analysis system was used firstly to outline the soma of each labelled neuron and then was focused on the overlying grains. Both the area of the neuronal profile, in $\mu \mathrm{m}^{2}$, as well as the number of grains present were determined and used to compute the intensity of labelling of each neuron, in grains $/ 1000 \mu \mathrm{m}^{2}$. The grains could be differentiated from the underlying melanin by their different plane of focus, as well as by using the program filter settings. The centre of each region of interest was identified at low magnification; neurons were then analysed in adjacent fields using a $100 \times$ water immersion lens (Leitz, Deerfield, IL, USA), counting from the centre towards the periphery of each region, until 30 cells had been counted. The intensity of the autoradiographic background was measured using a circular sample of $315 \mu \mathrm{m}^{2}$, the size chosen to correspond closely to the overall mean area of the neuronal profiles. Data from 30 near adjacent regions of this size overlying the crus cerebri were obtained from each section studied.

The intensities of labelling in each of the populations of neurons were compared with each other and to background labelling by using a one way factorial analysis of variance (ANOVA) with a significance level of $5 \%$. Grain density cell counts were collapsed into a mean value per region. A post hoc test (Fisher's predicted least squares difference) was used to calculate main effects for regions. The analyses were performed on a commercially available statistical software package (Statview 4.01, Abacus Concepts Inc, Berkeley, CA, USA). For figure presentation purposes, photographic images were digitised, arranged as a montage, and labelled using standard image editing software (Adobe Photoshop 4.0, Mountainview, CA, USA).

\section{Results}

NORTHERN ANALYSIS

Northern blot analysis of the DAT probe showed a single intense band in the lane corresponding to the substantia nigra, of approximately $5.0 \mathrm{~kb}$ in size. The lack of hybridisation to other brain regions confirmed the specificity of DAT to the substantia nigra.

IN SITU HYBRIDISATION

Inspection of film autoradiograms of control brains showed a high level of specific hybridisation to the DAT mRNA antisense probe across all regions studied in the ventral mesencephalon (figure 1A). No appreciable signal above background was noted in other midbrain regions, including the central periaqueductal grey substance (where identifiable). The apparent discrepancy in labelling intensity between the dorsal and ventral tier neuronal groups on autoradiograms is due to the lower cell density of the dorsal tier neurons than is the case in the ventral tier. There was no specific hybridisation in sections hybridised with a "sense" probe. Autoradiograms from Parkinson's disease 
Table 2 Mean grain density/1000 $\mu^{2}$ (mean (SEM)) for DAT $m R N A$ in regions of control and Parkinson's disease ventral mesencephalon

\begin{tabular}{lll}
\hline & Control & Parkinson's disease \\
\hline PN & $775.6(139.9)$ & $440.9(46.8)$ \\
SNpc-dorsal & $625.5(103)$ & $456(70.6) \dagger$ \\
SNpc-ventral & $760.1(66)$ & \\
PL & $691.6(112.7)$ & $466.9(83.4)$ \\
Background & $15.8(3)$ & $12(2)$
\end{tabular}

†This value represents the mean from a region including dorsal and ventral tiers of SNpc.

$\mathrm{PN}=$ paranigral nucleus; $\mathrm{SNpc}=$ substantia nigra pars compacta; $\mathrm{PL}=$ pars lateralis.

brains showed intense signal only in the medial ventral mesencephalon, in the region of the paranigral nucleus, with low but discernible hybridisation signal in the SNpc (fig 1B).

On control sections at a cellular level, virtually all neuromelanin containing cells expressed an intense hybridisation signal for DAT mRNA (fig 2). There was marked variation in the extent of labelling within each neuronal group studied, although virtually all melanised neurons with discernible cytoplasm showed significantly more labelling than background. Marked neuronal loss was evident in the SNpc of the Parkinson's disease sections, so much so as to obscure the boundaries between dorsal and ventral tier neuronal groups. Neuronal loss was minimal in the medial paranigral nucleus. In the surviving neurons, however, a modest to intense signal was apparent. In neurons in which Lewy bodies were identified, there was a marked reduction in hybridisation signal, compared with adjacent neurons (fig 2).

For quantitative analysis of hybridisation signal intensity in the control brains, four regions of the $\mathrm{SN}$ were studied: paranigral nucleus, dorsal and ventral tier neurons, and pars lateralis (fig 1 and table 2). Neurons from each region studied showed significant labelling compared with background $(p=0.002$ by ANOVA). Posthoc tests (Fisher's predicted least squares difference) showed no significant differences in signal between regions.

In the quantitative assessment of labelling intensity in the Parkinson's disease brains, dorsal and ventral tier neurons were analysed as a single region $(\mathrm{SNpc})$, as the anatomical boundary between dorsal and ventral tiers of the $\mathrm{SNpc}$ was indistinct. Neuronal labelling in each of these regions was significantly stronger than background ( $p<0.0001$ by ANOVA) but there was no significant differences between regions in these cases $(p=0.96)$.

In a separate quantitative analysis, the intensity of hybridisation signal was compared between regions of control and Parkinson's disease brains. For this analysis, a mean of combined dorsal and ventral tier cell counts was calculated to compare directly with $\mathrm{SNpc}$ of Parkinson's disease brains. The mean grain density $/ 1000 \mu \mathrm{m}^{2}$ for all regions was mean 720 (SEM 60.8) and 454 (SEM 37.3) for control and Parkinson's disease brains respectively, representing a significant reduction in mRNA expression in Parkinson's disease neurons compared with controls $(p=0.037)$. Hence, residual nigral neurons in Parkinson's disease brains expressed $63.1 \%$ of the DAT mRNA hybridisation intensity of neurons in the control brains. There was no significant difference between autoradiographic background signal in control and Parkinson's disease tissue $(\mathrm{p}=0.32)$. A pairwise comparison between regions of control versus disease brains (Fisher's predicted least squares difference) showed significant differences in labelling intensity between the paranigral nuclei $(p=0.037)$ as well as between neurons of the $\mathrm{SNpc}$ $(p=0.046)$, but not between neurons of the pars lateralis $(\mathrm{p}=0.14)$.

\section{Discussion}

Using in situ hybridisation histochemistry with a cRNA probe, we have determined the regional expression of DAT in selected neuronal subpopulations in the ventral mesencephalon of control and Parkinson's disease brains. In control brains, we found intense DAT gene expression in the ventral midbrain. Although there was no significant difference in mRNA concentrations among the different control midbrain regions studied, the mean grain density for DAT was stronger in the paranigral nucleus than in $\mathrm{SNpc}$ neurons. In the brains of patients with Parkinson's disease, we found an overall decrease in the intensity of DAT mRNA expression in the surviving dopaminergic neurons, confirming previous reports. ${ }^{15}{ }^{16}$ Additionally, we found a significant decline in DAT gene expression in neurons of the $\mathrm{SNpc}$ as well as the paranigral nucleus in Parkinson's disease brains, compared wiyh controls. These findings extend previous reports of regional DAT gene expression in human midbrain dopaminergic neurons, ${ }^{13-17}$ as well as providing new information regarding differential DAT mRNA distribution in defined subpopulations of nigral neurons in Parkinson's disease.

We found a trend toward higher mean silver grain density among neurons of the paranigral nucleus compared with the $\mathrm{SNpc}$ in controls, which argues against a significant role for DAT in rendering certain neuronal populations vulnerable to degeneration, as these neurons are known to be relatively spared in the disease process. Harrington et $a l^{16}$ also reported higher labelling of the paranigral nucleus than $\mathrm{SNpc}$, in accordance with our findings, although other studies found lower levels of hybridisation signal in paranigral nucleus than Snpc, ${ }^{15}$ paralleling the rodent data. ${ }^{10-12} 25$ These conflicting data may be explained in part by the lack of consensus in the literature regarding the internal structure of the three dopaminergic cell groups of the ventral mesencephalon (medial A10 cells, A1 1 cells of the SNpc, and A8 cells of the lateral reticular formation ${ }^{26}$ ) making the interpretation of anatomical studies in human substantia nigra difficult. Some detailed studies have delineated several subdivisions of the substantia nigra, ${ }^{12} 232728$ but there is little unanimity regarding the internal organisation of the A10 group. Interestingly, in rodents, there is substantial variability in the extent of DAT gene expression among subpopulations of the ventral tegmental area. ${ }^{10}$ In the present study, 
we distinguished the paranigral nucleus from other A10 cell group nuclei by the interspersing of oculomotor nerve fibres through this nucleus, although we could not always reliably distinguish the nucleus from the adjacent pars medialis of the A10 dopaminergic cell group. Hence our data are based on the recent attempts to standardise the nomenclature of nigral dopaminergic nuclei. ${ }^{18}$ As McRitchie et al point out ${ }^{29}$ however, such careful delineation of nigral subdivisions is neither trivial nor esoteric, as these nuclei have distinct projections as well as immunochemical characteristics, ${ }^{29-31}$ making this distinction has important implications for determining the selective vulnerability of midbrain dopaminergic neurons to degeneration..$^{32}$

In situ hybridisation studies in primates have shown high concentrations of DAT mRNA in the ventral tier neurons of the $\mathrm{SNpc}$, which are known to be most vulnerable to degeneration in Parkinson's disease, with less hybridisation in the dorsal tier and ventral tegmental area neurons. ${ }^{33}$ These findings indicate that the pattern of loss of dopaminergic cells is consistent with the degeneration of cells that contain the most mRNA for DAT, leading to the hypothesis that this rank order of expression might explain the selective vulnerability of ventral tier neurons to degeneration in Parkinson's disease, perhaps by exposing such neurons to an MPTP-like toxin. However, we found no significant difference in the intensity of DAT gene expression between dorsal and ventral tier neurons, which further argues against a significant role for the DAT in selective neuronal vulnerability in Parkinson's disease.

Our studies also show no significant regional variation in DAT gene expression in nigral neuron subpopulations from older subjects, in agreement with previous reports, ${ }^{17}$ suggesting that DAT expression does not confer an a priori vulnerability on dopaminergic neurons to a subsequent toxic insult.

Little information is available regarding the alterations in DAT gene expression in Parkinson's disease. ${ }^{15}{ }^{16} \mathrm{Uhl}$ et al ${ }^{15}$ found that $\mathrm{SNpc}$ dopaminergic neurons expressed $57 \%$ of the DAT mRNA hybridisation density of control brains, with a relative preservation of expression in neurons of the ventral tegmental area. These findings were confirmed in a subsequent report ${ }^{16}$ in agreement with the present study. Neither study, however, conducted a quantitative analysis to include the paranigral nucleus and pars lateralis of the substantia nigra, which are thought to have separate connectivities from other nigral regions. Interestingly, despite the relative preservation of paranigral nucleus neurons in Parkinson's disease, we found a significant decline in the expression of DAT in the paranigral nucleus of parkinsonian brains, compared with controls, in agreement with the report of Uhl et al..$^{34}$ Although neuronal loss has been reported to be more severe in the medial substantia nigra of demented patients with Parkinson's disease, ${ }^{35}$ none of our patients had evidence of cognitive decline.

The finding of reduced DAT mRNA in surviving dopaminergic neurons in Parkinson's disease is probably a reflection of ongoing cellular damage, although it has been suggested that it may be a compensatory change to increase the amount of dopamine in the synaptic cleft. ${ }^{16}$ Although the intensity of mRNA hybridisation signal is not necessarily a reflection of the translated protein, several immunochemical, receptor autoradiographic, and functional imaging analyses of DAT in control and Parkinson's disease striatum showed a topographical loss of immunoreactivity in a gradient from dorsal to ventral putamen, reflecting the greater loss of $\mathrm{SNpc}$ ventral tier neurons in Parkinson's disease. ${ }^{36-39}$

Interpretation of mRNA concentrations in human tissue must be considered in the light of significant technical issues, including both antemortem terminal events as well as postmortem interval. In our studies, control and Parkinson's disease brains were matched for postmortem interval. As tissue $\mathrm{pH}$ has been found to be strongly correlated with mRNA preservation in human postmortem brain, ${ }^{19}$ we excluded brains with $\mathrm{pH}$ less than 5.9 from analysis. Evidence for the specificity of hybridisation with our cRNA probe comes from its anatomical specificity (there is no labelling of the dorsal raphe, which contains a related amine transporter 5-hydroxytryptophan), as well as the fact that hDAT northern analysis parallels the in situ findings.

Our patients with Parkinson's disease were older than the controls, which may have had a bearing on the relative mRNA intensities between the two groups. Bannon et al have reported a significant down regulation of DAT mRNA with age, ${ }^{14}$ which was most prominent in $\mathrm{SNpc}$ ventral tier neurons. They showed a decline not only in the number of DAT mRNA positive cells but also a fall in the cellular abundance of DAT mRNA, suggesting that age related changes in DAT gene expression are not simply a reflection of neuronal loss. Interestingly, studies of the DAT gene have found several polymorphisms which may serve to mark allelic gene variants, which may account for individual differences in gene expression. ${ }^{40}$

Despite the extensive structural homology between the different members of the monoamine family, ${ }^{5}$ our data, using a sensitive technique, confirm that the DAT gene expression displays remarkable anatomic segregation. Moreover, the DAT receptor has remarkable pharmacological substrate specificity. ${ }^{41}{ }^{42}$ This has provided an opportunity for the development of DAT specific radioligands for in vivo striatal imaging in early Parkinson's disease, ${ }^{38}$ which may allow identification of presymptomatic cases and institution of neuroprotective therapy. In addition, the recent production of DAT knockout mice has also provided an excellent tool for the development of drugs for use in the management of disorders of dopaminergic function. ${ }^{41}$

Although it remains unclear whether the decrease in DAT gene expression in Parkinson's disease is a compensatory change to counteract the fall in synaptic dopamine concentrations, or whether it simply reflects 
neuronal dysfunction, our findings do not support the hypothesis that differential regional DAT gene expression underlies the selective vulnerability of nigral dopaminergic neurons in Parkinson's disease.

We thank Z Hollingsworth for excellent technical assistance, and S Augood, C Kosinski, and D Standaert for their helpful comments. TJC is the recipient of a National Parkinson Disease research fellowship. This work was supported by USPHS grant NS31579.

1 Gibb W, Lees A. Anatomy, pigmentation, ventral and dorsal subpopulations of the substantia nigra, and differential cell death in Parkinson's disease. $\mathcal{F}$ Neurol Neurosurg Psychiatry 1991;54:388-96.

2 Fearnley J, Lees A. Ageing and Parkinson's disease; substantia nigra regional selectivity. Brain 1991;114:2283301.

3 Hirsch E, Graybiel A, Agid Y. Melanized dopaminergic neurons are differentially susceptable to degeneration in Parkinson's disease. Nature 1988;334:345-8.

4 Amara SG, Kuhar MJ. Neurotransmitter transporters: recent progress. Ann Rev Neurosci 1993;16:73-93.

5 Giros B, Caron MG. Molecular characterization of the dopamine transporter. Trends in Pharmacological Sciences 1993;14:43-49.

6 Ritz MC, Lamb RJ, Goldberg SR, et al. Cocaine receptors on dopamine transporters are related to self-administration of cocaine. Science 1987;237:1219-23.

7 Javitch JA, Uhl GR, Snyder SH. Parkinsonism-inducing neurotoxin, N-methyl-4-phenyl-1,2,3,6tetrahydropyridine: characterization and localization of receptor binding sites in rat and human brain. Proc Nat Acad Sci (USA) 1984;81:4591-5.

8 Pifl C, Giros B, Caron MG. Dopamine transporter expression confers cytotoxicity to low doses of the parkinsoniansion confers cytotoxicity to low doses of the parkinsonian-
inducing neurotoxin 1-methyl-4-phenylpyridinium. $7 \mathrm{Neu}-$ inducing neurotoxin 1-me

9 Snyder S, D'Amato RJ. MPTP. A neurotoxin relevant to the pathophysiology of Parkinson's disease. Neurology 1986;36: $250-8$

10 Shimada S, Kitayama S, Walther D, et al. Dopamine transporter mRNA; dense expression in ventral midbrain neurons. Mol Brain Res 1992;13:359-62.

11 Cerruti C, Walther D, Kuhar MJ, et al. Dopamine transporter mRNA expression is intense in rat midbrain neurons and modest outside midbrain. Mol Brain Res 1993;18:181-6.

12 Augood SJ, Westmore K, McKenna PJ, et al. Co-expression of dopamine transporter mRNA and tyrosine hydroxylase mRNA in ventral mesencephalic neurons. Mol Brain Res 1993;20:328-34.

13 Hurd YL, Pristupa ZB, Herman MM, et al. The dopamine transporter and dopamine D2 receptor messenger RNAs are differentially expressed in limbic- and motor-related subpopulations of human mesencephalic neurons. Neurosubpopulations of hum

14 Bannon MJ, Poosch MS, Xia Y, et al. Dopamine transporter mRNA content in human substantia nigra decreases precipitously with age. Proc Nat Acad Sci (USA) 1992;89: 7095-9.

15 Uhl GR, Walther D, Mash D, et al. Dopamine transporte messenger RNA in Parkinson's disease and control substantia nigra neurons. Ann Neurol 1994;35:494-8.

16 Harrington KA, Augood SJ, Kingsbury AE, et al. Dopamine transporter (DAT) and synaptic vesicle amine transporter (VMAT2) gene expression in the substantia nigra of control and Parkinson's disease. Mol Brain Res 1996;36: 157-62.

17 Bannon MJ, Whitty CJ. Age-related and regional differences in dopamine transporter mRNA expression in human midbrain. Neurology 1997;48:969-77.

18 McRitchie DA, Hardman CD, Halliday GM. Cytoarchitectural distribution of calcium binding proteins in midbrain 1996;364:121-50.

19 Kingsbury AE, Foster OJF, Nisbet AP, et al. Tissue pH as an indicator of mRNA preservation in human postmortem brain. Mol Brain Res 1995;28:311-8.
20 Shimada S, Kitayama S, Lin C-L, et al. Cloning and expression of a cocaine-sensitive dopamine transporter complesion of a cocaine-sensitive dopamine tran
mentary DNA. Science 1991;254:576-8.

21 Sitzman JH, LeMotte PK. Rapid and efficient generation of PCR-derived riboprobe templates for in situ hybridization histochemistry. F Histochem Cytochem 1993;41:773-3776.

22 Landwehrmeyer GB, Standaert DG, Testa CM, et al. NMDA receptor subunit mRNA expression by projection neurons and interneurons in rat striatum. F Neurosci 1995; 15:5297-307.

23 Olszewski J, Baxter D. Cytoarchitecture of the human brainstem. Basel: Karger, 1954:52-8.

24 Bogerts B. A brainstem atlas of catecholaminergic neurons in man, using melanin as a natural marker. F Comp Neurol 1981;197:63-80.

25 Blanchard V, Raisman-Vozari R, Vyas S, et al. Differential expression of tyrosine hydroxylase and membrane dopamine transporter genes in subpopulations of dopaminergic neurons of the rat mesencephalon. Mol Brain Res 1994;22:29-40.

26 Dahlstrom A, Fuxe K. Evidence for the existence of monoamine-containing neurons in the central nervous system. I Demonstration of monoamines in cell bodies of brainstem neurons. Acta Physiol Scand (Suppl) 1964;232:155.

27 Hassler R. Zur Pathologie der Paralysis agitans und des postenzephalitischen Parkinsonismus. If Psychol Neurol 1938;48:387-476.

28 Braak H, Braak E. Nuclear configuration and neuronal types of the nucleus niger in the brain of the human adult. Human Neurobiology 1986;5:71-82.

29 McRitchie DA, Halliday GM. Calbindin D28k-containing neurons are restricted to the medial substantia nigra in humans. Neuroscience 1995;65:87-91.

30 Gerfen CR, Herkenham M, Thibault J. The neostriatal mosaic: II. Patch- and matrix-directed mesostriatal dopaminergic and non-dopaminergic systems. F Neuroscience 1987;7:3915-34.

31 Yamada T, McGeer PL, Baimbridge KG, et al. Relative sparing in Parkinson's disease of substantia nigra dopamine neurons containing calbindin-D28k. Brain Res 1990;526: 303-7.

32 McRitchie DA, Cartwright HR, Halliday GM. Specific A10 dopaminergic nuclei in the midbrain degenerate in Parkinson's disease. Exp Neurol 1997;144:202-13.

33 Haber SN, Ryoo H, Cox C, et al. Subsets of midbrain dopaminergic neurons in monkeys are distinguished by different levels of mRNA for the dopamine transporter: comparison with the mRNA for the D2 receptor, tyrosine hydroxylase and calbindin immunoreactivity. F Comp Neurol 1995;362:400-10.

34 Uhl GR, Hedreen JC, Price DL. Parkinson's disease: loss of neurons from the ventral tegmental area contralateral to therapeutic surgical lesions. Neurology 1985;35:1215-8.

35 Paulus W, Jellinger K. The neuropathologic basis of different clinical subgroups of Parkinson's disease. $\mathcal{7}$ Neuropathol Exp Neurol 1991;50:743-55.

36 Niznik HB, Fogel EF, Fassos FF, et al. The dopamine transporter is absent in Parkinsonian putamen and reduced in the caudate nucleus. $\mathcal{F}$ Neurochem 1991;56:192-8.

37 Ciliax BJ, Heilman C, Demchyshyn LL, et al. The dopamine transporter: immunochemical characterization and localization in brain. $\mathcal{F}$ Neurosci 1995;15:1714-23.

38 Booij J, Tissingh T, Boer GJ, et al. $\left[{ }^{123} \mathrm{I}\right] \mathrm{FP}-\mathrm{CIT}$ SPECT shows a pronounced decline of striatal dopamine transporter labelling in early and advanced Parkinson's disease. 7 Neurol Neurosurg Psychiatry 1997;62:133-40.

39 Miller GW, Staley JK, Heilman CJ, et al. Immunochemical analysis of dopamine transporter protein in Parkinson's disease. Ann Neurol 1997;41:530-9.

40 Vandenbergh D, Persico A. A human dopamine transporter cDNA predicts reduced glycosylation, displays a novel repetitive element and provides racially dimorphic Taq I RFLPs. Mol Brain Res 1992;15:161-6.

41 Giros B, Jaber M, Jones S, et al. Hyperlocomotion and indifference to cocaine and amphetamine in mice lacking the dopamine transporter. Nature 1996;379:606-12.

42 Kitayama S, Shimada S, Hongxia X, et al. Dopamine transporter site-directed mutations differentially alter substrate ransport and cocaine binding. Proc Natl Acad Sci (USA) 1992;89:7782-5. 